Journal of Computer Science 6 (10): 1130-1136, 2010

ISSN 1549-3636

(C) 2010 Science Publications

\title{
Linear Filtering for Optimized Approach in Satellite Image Enhancement
}

\author{
${ }^{1}$ Petcharat Pattanasethanon and ${ }^{2}$ Boonwat Attachoo \\ ${ }^{1}$ Department of Information Technology, Faculty of Information Technology, \\ King Mongkut's Institute of Technology Ladkrabang, No. 3 Moo 2, Chaloengkrung, \\ ${ }^{2}$ Department of Computer Engineering, Faculty of Engineering, \\ King Mongkut's Institute of Technology Ladkrabang, \\ Ladkrabang, 10520 Bangkok, Thailand
}

\begin{abstract}
Problem statement: For decades, several image enhancement techniques have been proposed. Although most techniques require profuse amount of advance and critical steps, the result for the perceive image are not as satisfied. Approach: In this study, we proposed a new method to enhance the satellite image which compares two procedures using two different kinds of filtering technique with an additional step in order to obtain the perceived image. In this new algorithm we first transform the color image into grayscale. The image is then preceded to the edge detection and brightness enhancement step using Laplacian and Sobel technique individually. Results: From the results, the Tenengrad averred that the enhancement result of the dimension and depth in the image were successfully classified. We also evaluate the image quality, adjusting by the PSNR and Tenengrad criterion which indicates that the proposed method shows dramatically increase in pixel distribution throughout the range of RGB. Conclusion: The result of this research is also beneficial in terms of geographical views due to the process which determined the difference appeared on each area. Eventually, this research also performed a comparison for the enhancement step mentioned in this study.
\end{abstract}

Key words: Image enhancement, sharpening, satellite image, edge detecting

\section{INTRODUCTION}

Image sharpening is one of several steps which enhances both the intensity and the edge of the images in order to obtain the perceive image. The step helps increase the resolution, the detail, as well as the sharpness of the image. In the early steps, before applying the data, image enhancement increases the difference between each object. As a consequence, the object and its edge were identical. In addition, image sharpening is eligible for emphasizing the individual location according to the scope of research. This convenient step constantly develops several techniques for the better results of the image (Welsh et al., 2002; Alparone et al., 2007). Recently, Wilscy and Nair (2008) proposed an image enhancement technique called fuzzy Technique in their research, however; the steps create conflict in the enhancement process. In this study, we proposed a continuous research from 'A new approach for color satellite image enhancement'
(Attachoo and Pattanasethanon, 2008) which compared and composed new steps and techniques in order to obtain the perceived image. Generally, the principle for image filtering method and edge detection can be done by several techniques. Firstly, signal reduction is required to emphasize the edge and brighten the image. In this case, high pass filter is used to filter the signal as well as to detect the edges from the original image. Hence, the solution for this process is the total of the original image and the edge as the equation below:

$\mathrm{f}_{\mathrm{s}}\left(\mathrm{x}_{\mathrm{i}}, \mathrm{y}_{\mathrm{i}}\right)=\mathrm{f}\left(\mathrm{x}_{\mathrm{i}}, \mathrm{y}_{\mathrm{i}}\right)+\Omega \mathrm{F}\left(\mathrm{f}\left(\mathrm{x}_{\mathrm{i}}+\mathrm{y}_{\mathrm{i}}\right)\right)$

Where:

$\mathrm{f}\left(\mathrm{x}_{\mathrm{i}}, \mathrm{y}_{\mathrm{i}}\right)=$ The original pixel value at the coordinate $\left(\mathrm{x}_{\mathrm{i}}, \mathrm{y}_{\mathrm{i}}\right)$

$\mathrm{F}() \quad=$. The high pass filter

$\Omega \quad=$ A tuning parameter which is greater or equal to zero

$\mathrm{f}_{\mathrm{s}}\left(\mathrm{x}_{\mathrm{i}}, \mathrm{y}_{\mathrm{i}}\right)=$ The sharpened pixel at the coordinate $\left(\mathrm{x}_{\mathrm{i}}, \mathrm{y}_{\mathrm{i}}\right)$

Corresponding Author: Petcharat Pattanasethanon, Department of Information Technology, Faculty of Information Technology, King Mongkut's Institute of Technology Ladkrabang, No. 3 Moo 2, Chaloengkrung, Ladkrabang, 10520 Bangkok, Thailand 
The value represents $\Omega$ as the perspective degree of sharpness, the higher the $\Omega$ the more sharpened is the image. Another well known technique which enhances blur images is called Unsharp Masking (UM) technique. The solution of this technique begins by subtracting the original image with the blur image.

In the other words, subtract low pass filter from the input image. This results for the output image which emphasizes on the detail and sharpness (Xu and Wang, 2009).

Generally, blurred images occur by several low pass filtering in the image. Hereby was the equation for Unsharp Masking technique:

$$
\mathrm{f}_{\mathrm{s}}\left(\mathrm{x}_{\mathrm{i}}, \mathrm{y}_{\mathrm{i}}\right)=\mathrm{f}\left(\mathrm{x}_{\mathrm{i}}, \mathrm{y}_{\mathrm{i}}\right)-\mathrm{f}_{\mathrm{b}}\left(\mathrm{x}_{\mathrm{i}}, \mathrm{y}_{\mathrm{i}}\right)
$$

Where:

$f_{s}(x, y)=$ The sharpened image obtained by unsharp masking

$f_{b}(x, y)=$ The blurred version of $f\left(x_{i}, y_{i}\right)$

According to the second equation, increase in sharpness is eligible by using high boost filter (Gonzales and Woods, 2002). The relations between two equations were as shown below:

$$
\mathrm{f}_{\mathrm{sh}}\left(\mathrm{x}_{\mathrm{i}}, \mathrm{y}_{\mathrm{i}}\right)=\mathrm{A} * \mathrm{f}\left(\mathrm{x}_{\mathrm{i}}, \mathrm{y}_{\mathrm{i}}\right)-\mathrm{f}_{\mathrm{b}}\left(\mathrm{x}_{\mathrm{i}}, \mathrm{y}_{\mathrm{i}}\right)
$$

or:

$$
\mathrm{f}_{\mathrm{sh}}\left(\mathrm{x}_{\mathrm{i}}, \mathrm{y}_{\mathrm{i}}\right)=(\mathrm{A}-1)+\mathrm{f}_{\mathrm{h}}\left(\mathrm{x}_{\mathrm{i}}, \mathrm{y}_{\mathrm{i}}\right)
$$

A $=$ A variable which is greater or equal to 1

$\mathrm{f}_{\mathrm{sh}}\left(\mathrm{x}_{\mathrm{i}}, \mathrm{y}_{\mathrm{i}}\right)=$ The high boost sharpened image

$\mathrm{f}_{\mathrm{b}}\left(\mathrm{x}_{\mathrm{i}}, \mathrm{y}_{\mathrm{i}}\right)=$ The low pass filter of $\mathrm{f}\left(\mathrm{x}_{\mathrm{i}}, \mathrm{y}_{\mathrm{i}}\right)$

$\mathrm{f}_{\mathrm{h}}\left(\mathrm{x}_{\mathrm{i}}, \mathrm{y}_{\mathrm{i}}\right)=$ The high pass filter of $\mathrm{f}\left(\mathrm{x}_{\mathrm{i}}, \mathrm{y}_{\mathrm{i}}\right)$

The previous methods stated can work under certain circumstances, meanwhile, it is inaccurate to use the method in case of low contrast image or low resolution image (Chen et al., 2006). Another method was proposed lately on satellite image enhancement (Attachoo and Pattanasethanon, 2008), which proposed an additional step by enhancing the brightness of the image before working on edge detection. However, the process shows no statistical results in the research. Therefore, the quality evaluation was still un-identical in order to compare the results. In this study we proposed a novel approach satellite image sharpening which continues from our previous research (Attachoo and Pattanasethanon, 2008). We developed new algorithms in intensity evaluation and compare its quality with its original version. The process were composed of image brightness, edge detection and the standard deviation of the image intensity performed by the Peak Signal to Noise Ratio (PSNR) and Tenengrad (Moustafa and Badawy, 2007; Ying et al., 2008), respectively (Welsh et al., 2002).

\section{MATERIALS AND METHODS}

A new approach sharpening model: The new approach sharpening model in this research begins with brightness enhancement. Then follow by edge detection, using the equation below:

$\mathrm{G}_{\mathrm{sc}}\left(\mathrm{x}_{\mathrm{i}}, \mathrm{y}_{\mathrm{i}}\right)=\mathrm{f}\left(\mathrm{x}_{\mathrm{i}}, \mathrm{y}_{\mathrm{i}}\right)-\nabla^{2}\left\{\mathrm{~T}\left[\mathrm{f}\left(\mathrm{x}_{\mathrm{i}}, \mathrm{y}_{\mathrm{i}}\right)\right]\right\}$

Where:

$\mathrm{G}_{\mathrm{sc}}(\mathrm{x}, \mathrm{y})=$ The new sharpened image

$\mathrm{f}\left(\mathrm{x}_{\mathrm{i}}, \mathrm{y}_{\mathrm{i}}\right)=$ The original image

$\mathrm{T}[]=$. The function for contrast enhancement

$\nabla^{2}=$ Laplacian edge detection

The algorithm for this new sharpening technique from Eq. 5 was as shown in Fig. 1. Another test is performed in order to compare the results with the first technique using Eq. 1. We performed an edge detection process with Laplacian and Sobel. The algorithm of this technique was processed according to the sharpening model, which on the first stage, the color information of the digital image are transformed into grayscale image. Then, linear contrast stretch approaches to enhance the brightness of the image. The image is then edge detected by finding the second derivative of the Laplacial or Sobel method. Finally, increase the sharpness of the image by subtracting the result with the original image.

Color model and image intensity consideration: In 1913, the Commission Internationale de I'Eclairage (CIE-the International Commission on Illumination) developed the original standard for visible spectrum (Gonzales and Woods, 2002). Certain colors were naturally visible formed by the combination of three main colors which were red, green and blue. As a consequence, the color model or CIE chromaticity diagram was proposed. While considering the video color image, the color intensity of the video image appears by the pixel performance. Each pixel components were composed of RGB. According to the RGB color model, the weight for the three colors; red, green and blue, varies due to the mixture of the intensity and luminance. The relation of the grayscale brightness and RGB on account of the CIE chromaticity 
diagram is correlated. The coefficient equals to the weight multiply by its color array (Berns, 2000) as the equation below:

$$
\begin{aligned}
\mathrm{f}_{\mathrm{gr}}(\mathrm{x}, \mathrm{y}) & =0.2989 * \mathrm{f}_{\mathrm{R}}(0,0,1)+0.5870 * \mathrm{f}_{\mathrm{G}}(0,0,2) \\
& +0.1140 * \mathrm{f}_{\mathrm{B}}(0,0,3)
\end{aligned}
$$

Where:

$$
\begin{aligned}
\mathrm{f}_{\mathrm{gr}}(\mathrm{x}, \mathrm{y}) & =\text { The grayscale image } \\
\mathrm{f}_{\mathrm{R}}(0,0,1), \mathrm{f}_{\mathrm{G}}(0,0,2), \mathrm{f}_{\mathrm{B}}(0,0,3)= & \text { The components of the } \\
& \text { red, green and blue } \\
& \text { image, respectively }
\end{aligned}
$$

However, in the cases which we transform the grayscale image into RGB image, influence conflicts to the CIE chromaticity diagram.

Hue, Saturation and Value (HSV) color model was proposed by Smith (1978). This model was proposed to be an alternative choice, which influences convenience in terms of color usage instead of using only the three main colors (RGB). The HSV model performs better details artistically and it is easier to understand the image by sightseeing. The details performed in the color image in each pixel were composed of the RGB value.

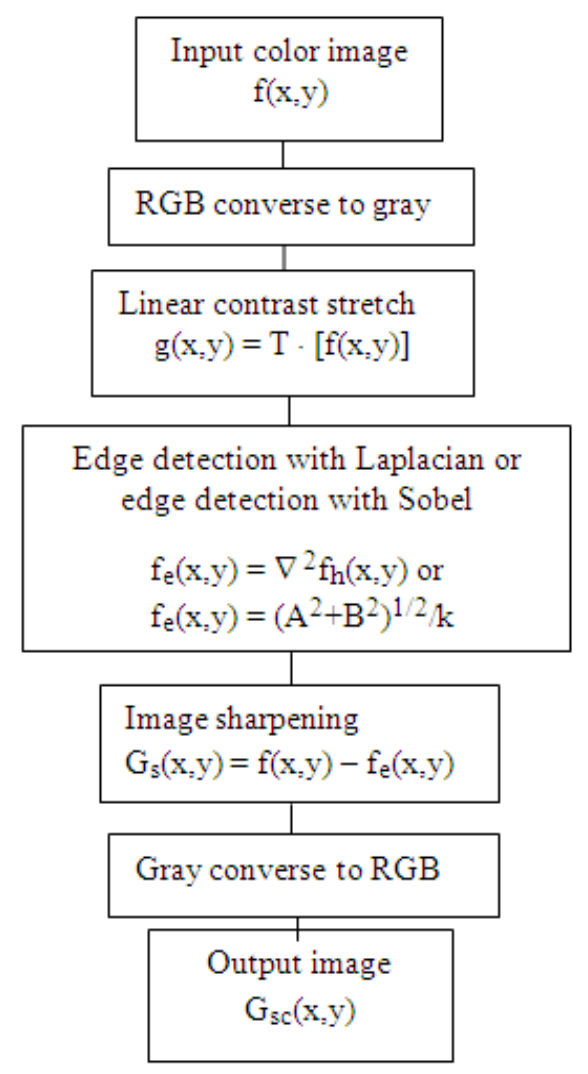

Fig. 1: Algorithm diagram for satellite image sharpening
Figure 2 shows the correlation of Hue, Saturation and Brightness value, respectively. Hue is the pureness intensity of colors, it can be measure in $0-360^{\circ}$ angle. The three main colors were $60^{\circ}$ difference. $\mathrm{S}$ refers to saturation, the dark intensity of color which values between 0-100. The color increases its darkness as the saturation value raise up. Brightness value or $\mathrm{V}$ is the value between $0-100$ of brightness intensity. The hue of a point is determined by an angle from some reference point.

RGB to HSV conversion: The obtainable HSV colors lie within a triangle whose vertices are defined by the three primary colors in RGB space (Fig. 3).

The hue of the point $\mathrm{P}$ is the measured angle between the connecting $\mathrm{P}$ to the triangle center and line connecting RED point to the center of the triangle. The saturation of the point $\mathrm{P}$ is the distance between $\mathrm{P}$ and triangle center. The value (intensity) of the point $\mathrm{P}$ represents height on the line perpendicular to the triangle and passing through its center. The grayscale points are situated onto the same line. The conversion formula was as follows:

$$
\begin{aligned}
& H=\cos ^{-1}\left\{\frac{\frac{1}{2}[(R-g)+(R-b)]}{\sqrt{(R-g)^{2}+(R-b)(G-b)}}\right\} \\
& S=1-\frac{3}{R+G+B}[\min (R, G, B)] \\
& V=\frac{1}{3}(r+g+b)
\end{aligned}
$$

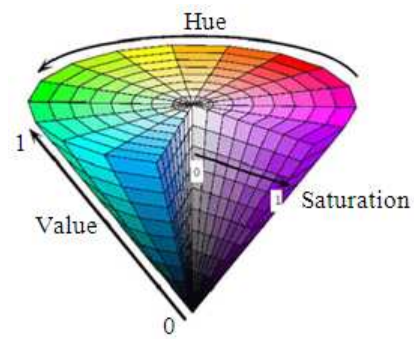

Fig. 2: The relationship between color model in RGB and HSV system

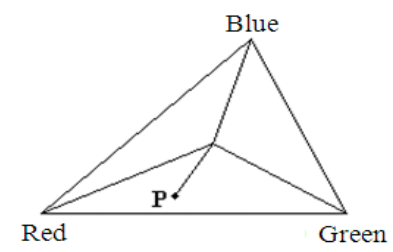

Fig. 3: Obtainable HSV color from RGB color space 
HSV to RGB conversion: Conversion from HSV space to RGB space is more complex. Particularly, given to the nature of the hue information, we obtained different formula for each sector of the color triangle.

Red-green sector: for $0^{\circ}<\mathrm{H} \leq 120^{\circ}$ :

$$
\mathrm{b}=\frac{1}{3}(1-\mathrm{s}), \mathrm{r}=\frac{1}{3}\left[1-\frac{\mathrm{S} \cos \mathrm{H}}{\cos \left(60^{\circ}-\mathrm{H}\right)}\right], \mathrm{g}=1-(\mathrm{r}-\mathrm{b})
$$

Green-blue sector: for $120^{\circ}<\mathrm{H} \leq 240^{\circ}$ :

$$
\mathrm{r}=\frac{1}{3}(1-\mathrm{s}), \mathrm{g}=\frac{1}{3}\left[1+\frac{\mathrm{S} \cos \mathrm{H}}{\cos \left(60^{\circ}+\mathrm{H}\right)}\right], \mathrm{b}=1-(\mathrm{r}+\mathrm{g})
$$

Blue-red sector: for $240^{\circ}<\mathrm{H} \leq 360^{\circ}$ :

$$
\mathrm{g}=\frac{1}{3}(1-\mathrm{s}), \mathrm{b}=\frac{1}{3}\left[1+\frac{\mathrm{S} \cos \mathrm{H}}{\cos \left(60^{\circ}-\mathrm{H}\right)}\right], \mathrm{r}=1-(\mathrm{g}+\mathrm{b})
$$

Linear contrast stretch: This technique modifies the linear contrast stretch that was related to the value transformation of the brightness part of the image. It can be measured by the lowest value of the brightness contrast (grey level $=0$ ) to the highest value of the brightness contrast (grey level $=255$ ) to full the grey scale level. A brightness value of between 0-255 would be spread out and could be calculated with the equation below (Xu and Wang, 2009):

$$
g(x, y)=T[f(x, y)]
$$

If:

$\mathrm{g}(\mathrm{x}, \mathrm{y})=$ A brightness value of the area in the image at the exit

$f(x, y)=$ The brightness value of the area at the entrance

$\mathrm{T}=$ The function for the linear transformation

Edge detection: Edge detection is generally to define edges of the object inside the image. Edges can be found when the difference between luminance intensity from one point to the other appears. Practically, the more difference of light luminance, the edges are easier to define. In contrast, the lesser the difference the harder the edges can be define. Edge detection evaluates the brightness of each area with difference luminance. The filtering technique in this research was performed by using Laplacian and Sobel technique, due to its evaluation capacity which filters the image constantly and spontaneously. According to the past articles, they have been several discoveries represented in this field for over 40 years which the practical filtering technique are for instance gradient edge detection (1st derivative), zero crossing (2nd derivative), Laplacian Of Gaussian (LOG) and Gaussian edge detection (Sharifi et al., 2002). However, the recent ones were such as fuzzy method which deals with several complications and time consuming throughout the procedure (Kang and Wang, 2007) and another one was Gradient edge detection which filtered by computing the first derivative from the image. This technique faces several limitations i.e., adding thick layers to the edges due to its slope from the result of first derivative computation at different grayscale level; from highest to lowest or conversely opposition. In addition, the result of the first derivative leads to the incorrect grayscale level from the original. By computing the second derivative we obtain Laplacian method which was proven to have a better filtering result than the first method. This method separates the thin layers of the area and determines the differences of each pixel accurately. Therefore, the benefits of the Laplacian method completely outweigh those of gradient edge detection (Jain, 1988) the equation is as follows:

$\nabla^{2} f(x, y)=\frac{\partial^{2} f(x, y)}{\partial x^{2}}+\frac{\partial^{2} f(x, y)}{\partial y^{2}}$

From Eq. 14, the equation can be written in the $\mathrm{x}, \mathrm{y}$ function form as below:

$$
\begin{aligned}
\nabla^{2} \mathrm{f}(\mathrm{x}, \mathrm{y})= & \mathrm{f}(\mathrm{x}+1, \mathrm{y})+\mathrm{f}(\mathrm{x}-1, \mathrm{y})+\mathrm{f}(\mathrm{x}, \mathrm{y}+1) \\
& +\mathrm{f}(\mathrm{x}, \mathrm{y}-1)-4 \mathrm{f}(\mathrm{x}, \mathrm{y})
\end{aligned}
$$

For color images, using Laplacian process edge detection would detect each image component separately. Nevertheless, in some cases, the side effects were found on certain areas during the procedure or errors produced in certain rows and columns of the image. Thus, edge detection with Laplacian also has limitations as well. The kernel is another important circumstance to determine the most suitable kernel for the pixels filtered. Sobel is considered a well replacement of Laplacian filter. According to the Sobel characteristics, the total factor of the filter equals to zero which in certain cases it can reduce the overflow problematic (Yan et al., 2002). In digital images $\mathrm{f}(\mathrm{x}, \mathrm{y})$, Sobel operator, $\mathrm{S}(\mathrm{x}, \mathrm{y})$ in the convolution procedure and image were set to as the coefficients below:

$$
\begin{aligned}
A= & {[f(x-1, y+1)+2 f(x-1, y)+f(x-1, y-1)] } \\
& -[f(x-1, y+1)+2 f(x+1, y)+f(x+1, y-1)]
\end{aligned}
$$




$$
\begin{aligned}
& B=[f(x-1, y-1)+2 f(x, y-1)+f(x, y-1)] \\
& +[f(x+1, y-1)+2 f(x, y+1)+f(x+1, y+1)] \\
& \mathrm{S}_{\mathrm{m}}(\mathrm{x}, \mathrm{y})=\left(\mathrm{A}^{2}+\mathrm{B}^{2}\right)^{1 / 2}
\end{aligned}
$$

Where:

$\mathrm{A}=$ The elements in the first and third column $\mathrm{B}=$ The elements in the first and third row

After estimating (18), we obtain Eq. 19:

$$
\mathrm{S}_{\mathrm{m}}(\mathrm{x}, \mathrm{y}) \approx \mathrm{A}+\mathrm{B}
$$

From (16) and (17), the equations developed Sobel kernel which filters the edge of the image as of Fig. 4. Each components placed in the kernel were the weighting factor of the output pixel.

The edge detection evaluation S (x.y) and position $(\mathrm{x}, \mathrm{y})$ may need a suitable threshold level. However, specifying the threshold level may perform lost of edge in certain parts of the image which the level is lower than the threshold. Separating the overflow information would affect the value which we can reduce the results by dividing by the scale-factor. As a consequence, the grayscale evaluation obtains a better result than binary evaluation, as the equation expressed below:

$\mathrm{S}_{\mathrm{m}}(\mathrm{x}, \mathrm{y})=\left(\mathrm{A}^{2}+\mathrm{B}^{2}\right)^{1 / 2} / \mathrm{k}$

$\mathrm{S}_{\mathrm{m}}(\mathrm{x}, \mathrm{y}) \approx(\mathrm{A}+\mathrm{B}) / \mathrm{k}$

When, $\mathrm{k}$ refers to a constant of the scale-factor.

Image quality evaluation: In this research, result image can be evaluated with two characteristics, distortion and sharpness. According to the distortion evaluation, adjusting errors are required, by computing the Mean Square Error (MSE). Mean square error has been the performance metric in lost performance. Peak Signal to Noise Ratio (PSNR) adjusts the quality of the image which the higher the PSNR refers to the better quality is the image (Moustafa and Badawy, 2007). The formula for MSE and PSNR are:

$$
\begin{aligned}
\mathrm{MSE}=\frac{\sum_{\mathrm{MN}\left[\mathrm{I}_{1}(\mathrm{~m}, \mathrm{n})-\mathrm{I}_{2}(\mathrm{~m}, \mathrm{n})\right]^{2}}}{\mathrm{M} . \mathrm{N}} \\
\begin{array}{rll}
1 & 0 & -1 \\
2 & 0 & -2 \\
1 & 0 & -1
\end{array}
\end{aligned}
$$

(a)
Fig. 4: Sobel operator mask (a) weight factor element in A (b) weight factor element in B
The MSE expression is generally referred to the absolute error equation because the former error is analytically tractable. The most common error in image processing is the normalized brightness of the image. Alparone et al. (2007) In the previous equation, M and $\mathrm{N}$ are the number of rows and columns of the input image, respectively. Then, all the blocks would compute the PSNR using the following equation:

$$
\mathrm{PSNR}=10 \log _{10}\left[\frac{\mathrm{R}^{2}}{\mathrm{MSE}}\right]
$$

In the above mentioned equation, $\mathrm{R}$ is the maximum fluctuation in the input image data type. For the second evaluation, the sharpness evaluation, we compute by using Tenengrad criterions; TEN, to evaluate the sharpness of the image. The fundamental idea to measure the differences and evaluating the sharpness is determined by the gradient of edge:

$$
\text { Ten }=\sum_{i, j}\left[f_{x}^{2}(i, j)+f_{y}^{2}(i, j)\right]
$$

While:

$$
\sqrt{f_{x}^{2}(i, j)+f_{y}^{2}(i, j)} \geq T
$$

where the horizontal and vertical gradients $f_{x}^{2}(i, j)$ and $\mathrm{f}_{\mathrm{y}}{ }^{2}(\mathrm{i}, \mathrm{j})$ are obtained by the Sobel filters and $\mathrm{T}$ is the threshold. Eventually, the image with the higher TEN obtained the higher sharpness as well (Yan et al., 2002).

\section{RESULTS}

The images tested in this research were performed in Fig. 5 which was express in the numerical form of satellite image, LANDSAT 7 ETM + system. The image represents false color image with band 4, 3 and 2, recorded and issued on January 22, 2002, covering the North Eastern area of Thailand.

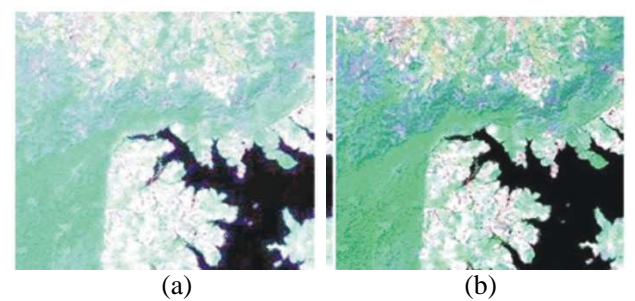

Fig. 5: Satellite image (a) original image (b) enhanced image 
One dot expresses 8 bits/pixel of information. LANDSAT 7 ETM + satellite contained $30 \mathrm{~m}$ of spatial resolution in band 1-5 and band 7, exceptional for band 6 , which contains $60 \mathrm{~m}$ of spatial resolution. The final band, panchromatic band contains $15 \mathrm{~m}$ of spatial resolution and save information in band sequential form.

\section{DISCUSSION}

In order to compare the results accurately, the experiment was divided into two groups according to the algorithms expressed in Eq. 1 and 5, respectively. Each group would be processed with different types of edge detection, Laplacian or Sobel, to compare the results.

Figure $6 a$ and $6 b$ represents the final step which includes subtracting the edge detected image from the original image. As a consequence, the MSE of the output image and the input image values at 0.66 and 0.52 , respectively (Table 1). Both values were considered no significant. However, the covariance of both color images remains at 0.91 and 0.92 which verify that the color is not distortion at the stable stage. In this study, we determined the total difference by comparing the PSNR between the original image and the output image, which the PSNR values at 25.67 and $28.95 \mathrm{~dB}$, respectively.

Table 1: Comparison of image sharpened quality

\begin{tabular}{llllll}
\hline Output & $\mathrm{MSE}$ & $\mathrm{R}^{2}$ & PSNR & TEN & Running time \\
\hline Image 1 & 0.660 & 0.91 & 25.67 & 43611 & 15 \\
Image 2 & 0.520 & 0.92 & 28.95 & 45118 & 20 \\
Image 3 & 0.254 & 0.94 & 27.76 & 91029 & 50 \\
Image 4 & 0.238 & 0.96 & 29.57 & 103712 & 60 \\
\hline
\end{tabular}

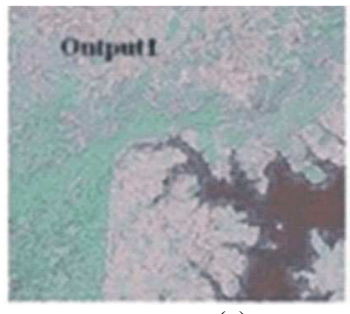

(a)

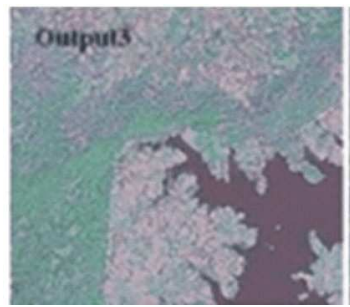

(c)

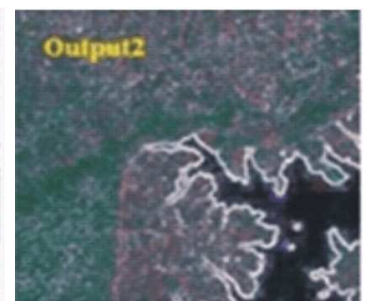

(b)

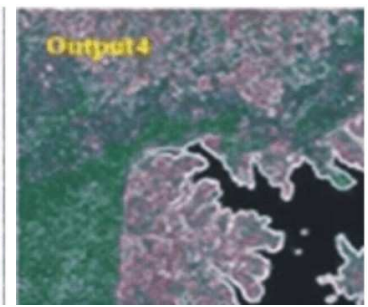

(d)
Fig. 6: The sharpened images in total
Practically, the PSNR which values higher than $30 \mathrm{~dB}$ is invisible for human sight to analyze the color distortion between two images (Moustafa and Badawy, 2007). As a comparison result, the PSNR indicate that proceeding with the Sobel technique for edge detect obtained a better result at the output image. As well as the pixel distribution result under the RGB axis of the color image, Fig. $6 a$ and $6 \mathrm{~b}$ show no change in pixel distribution with the input image.

The experimental result with the new algorithm procedure as describe in Eq. 5, Fig. 5a as the original image and Fig. 5b is the result of color to grayscale transformation and brightness enhancement with linear contrast stretch, respectively. Finally, Fig. $6 \mathrm{c}$ and d show the result of the difference between the edges detected image and the original image.

According to the result of the new algorithm, we compute the MSE of Fig. 6c and 6d which values at 0.254 and 0.238 , respectively. Simultaneously, $\mathrm{R}^{2}$ values at 0.94 and 0.96 , respectively. Comparing with the former procedure, we obtain different result due to the additional step which is linear contrast stretch. The comparisons also include the PSNR of Fig. 6c and c as well, which values at 27.76 and $29.56 \mathrm{~dB}$, respectively. According to the PSNR result, the PSNR was much higher than $30 \mathrm{~dB}$, this indicate that color distortion is invisible. However, the Tenengrad computed perform considerably high value which is 91029 and 103712, respectively. This also indicates that it obtained higher quality. This phenomenon appears on account of the step which include linear contrast stretch. The additional step increases the level of saturation in the color image while enhancing the image simultaneously (Fig. 7).

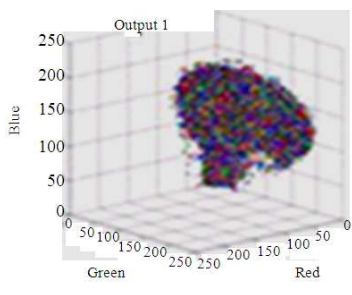

(a)

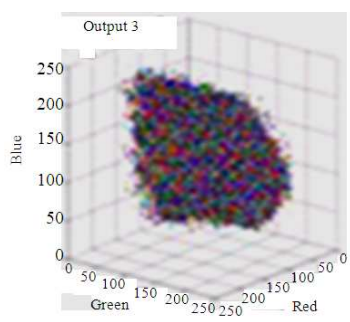

(c)

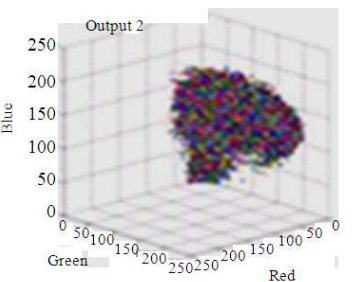

(b)

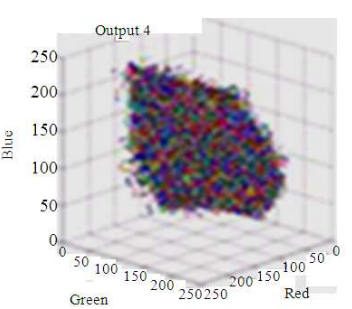

(d)
Fig. 7: Pixel distribution for all the enhanced images 
Finally, this is the most suitable step for satellite image to obtain the perceived image because in geographical images, the area, the height and the dept are required to be classified.

Running time: The running time of the algorithm for one image can range from 15-60 sec on a AMD Turion 64-2.0 GHz, CPU using optimized MATLAB cod. Version 7.6 (R2008a) "Transferring Color to Grayscale Images".

\section{CONCLUSION}

The novel sharpening method procedure was experimented with additional steps. We first transform the image from color image into grayscale, then begin edge detecting with Laplacial technique. The edge detected image is then subtracted from the original image. The result of the image was as expected; the Tenengrad criterion indicates its high quality after the enhancement process was performed. For satellite images, this technique is expedient. The result image manifested the difference in certain areas, the dimension and the depth as well. Simultaneously, the result image classifies the items and objects perspicuously, as well as superimposing the color saturation.

In the formal way, the final step which subtract the original image with the edge detected image benefits the scenery of the image and color is enhanced. In conclusion, the prototype enhancement procedure and the novel enhancement procedure indicates that proceeding with Laplacial filtering technique provide a more expedient result than of the Soble filtering technique.

\section{REFERENCES}

Alparone, L., L. Wald, J. Chanussot, C. Thomass and P. Gamba et al., 2007. Comparison of pansharpening algorithms: Outcome of the 2006 GRS-S data-fusion contest. IEEE Trans. Geosci. Remote Sens., 45: 3012-3021. DOI: 10.1109/TGRS.2007.904923

Attachoo, B. and P. Pattanasethanon, 2009. A new approach for colored satellites image enhancement. Proceeding of the IEEE International Conference on Robotics and Biomimetics, Feb. 22-25, IEEE Xplore Press, Bangkok, pp: 1365-1370. DOI: 10.1109/ROBIO.2009.4913199

Berns, R.S., 2000. Billmeyers and Saltzman's Principles of Color Technology. 3rd Edn., John Wiley and Sons, New York, ISBN: 13: 9780471194590, pp: 304.
Chen, Z.Y., B.R. Abidi, D.L. Page and M.A. Abidi, 2006. Gray Level Grouping (GLG): An automatic method for optimized image contrast enhancementPart I: The basic method. IEEE Trans. Image Process., 15: 2290-2302. DOI: 10.1109/TIP.2006.875204

Gonzales, R.C. and R.E. Woods, 2002. Digital Image Processing. 2nd Edn., Prentice Hall, USA., ISBN: 10: 0130946508, pp: 793.

Jain, A.K., 1988. Fundamentals of Digital Image Processing. US Edn., Prentice Hall, USA., ISBN: 10: 0133361659, pp: 569.

Kang, C.C. and W.J. Wang, 2007. A novel edge detection method based on the maximizing objective function. Patt. Recog., 40: 609-618. DOI: 10.1016/j.patcog.2006.03.016

Moustafa, K.A. and W. Badawy, 2007. (Color/gray) image in color covers hiding using modification of spatial domain hiding method. Future Generat. Commun. Network., 1: 56-61. DOI: 10.1109/FGCN.2007.1

Sharifi, M., M. Fathy and M.T. Mahmoudi, 2002. A classified and comparative study of edge detection algorithms. Proceeding of the International Conference on Information Technology: Coding and Computing, Apr. 8-10, IEEE Computer Society, Washington DC., USA., pp: 117-120.

Smith, A.R., 1978. Color gamut transform pairs. Comput. Graph., 12: 12-19.

Welsh, T., M. Ashikhmin and K. Mueller, 2002. Transferring color to greyscale images. ACM Trans. Graph., 21: 277-280. DOI: $10.1145 / 566570.566576$

Wilscy, M. and S.M. Nair, 2008. A new method for sharpening color images using fuzzy approach. Lecture Notes Comput. Sci., 5112: 65-74. DOI: 10.1007/978-3-540-69812-87

Xu, D. and R. Wang, 2009. An improved FoE model for image deblurring. Int. J. Comput. Vis., 81: 167-171. DOI: $10.1007 / \mathrm{s} 11263-008-0155-3$

Yan, X., L. Kai-Yang, Y. Xuan-Dong, W.X. Fang and Z.X. Lin et al., 2002. The application of the edge sharpening operator to the breast near infrared. Wuhan Univ. J. Nat. Sci., 7: 421-425. DOI: 10.1007/BF02828241

Ying, L., N.T. Ming and L.B. Keat, 2008. A wavelet based image sharpening algorithm. Proceeding of the 2008 International Conference on Computer Science and Software Engineering, Dec. 12-14, IEEE Computer Society, Washington DC., USA., pp: 1053-1056. DOI: 10.1109/CSSE.2008.1631 